\title{
Marine sediments illuminate Chlamydiae diversity and evolution
}

\author{
Jennah E. Dharamshi ${ }^{1}$, Daniel Tamarit ${ }^{1} \dagger$, Laura Eme ${ }^{1} \dagger$, Courtney Stairs ${ }^{1}$, Joran Martijn ${ }^{1}$, Felix \\ Homa $^{1}$, Steffen L. Jørgensen ${ }^{2}$, Anja Spang ${ }^{1,3}$, Thijs J. G. Ettema ${ }^{1,4 *}$ \\ ${ }^{1}$ Department of Cell and Molecular Biology, Science for Life Laboratory, Uppsala University, \\ SE-75123 Uppsala, Sweden \\ ${ }^{2}$ Department of Earth Science, Centre for Deep Sea Research, University of Bergen, N-5020 \\ Bergen, Norway \\ ${ }^{3}$ Department of Marine Microbiology and Biogeochemistry, NIOZ Royal Netherlands Institute \\ for Sea Research, and Utrecht University, NL-1790 AB Den Burg, The Netherlands \\ ${ }^{4}$ Laboratory of Microbiology, Department of Agrotechnology and Food Sciences, Wageningen \\ University, NL-6708 WE Wageningen, The Netherlands. \\ $\uparrow$ These authors contributed equally \\ * Correspondence to: Thijs J. G. Ettema, Email: thijs.ettema@wur.nl
}


1 The bacterial phylum Chlamydiae, which is so far comprised of obligate symbionts of

2 eukaryotic hosts, are well-known as human and animal pathogens ${ }^{1-3}$. However, the

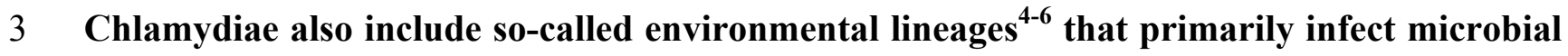

4 eukaryotes $^{7}$. Studying environmental chlamydiae, whose genomes display extended

5 metabolic capabilities compared to their pathogenic relatives ${ }^{8-10}$ has provided first insights

6 into the evolution of the pathogenic and obligate intracellular lifestyle that is characteristic

7 for this phylum. Here, we report an unprecedented relative abundance and diversity of

8 novel lineages of the Chlamydiae phylum, representing previously undetected, yet

9 potentially important, community members in deep marine sediments. We discovered that

10 chlamydial lineages dominate the microbial communities in the Arctic Mid-Ocean Ridge ${ }^{11}$,

11 which revealed the dominance of chlamydial lineages at anoxic depths, reaching relative

12 abundances of up to $43 \%$ of the bacterial community, and a maximum diversity of 163

13 different species-level taxonomic unit. Using genome-resolved metagenomics, we

14 reconstructed 24 draft chlamydial genomes, thereby dramatically expanding known

15 interspecies genomic diversity in this phylum. Phylogenomic and comparative analyses

16 revealed several deep-branching Chlamydiae clades, including a sister clade of the

17 pathogenic Chlamydiaceae. Altogether, our study provides new insights into the diversity,

18 evolution and environmental distribution of the Chlamydiae. 
24 During a previous metagenomics study aimed at exploring microbial diversity of deep marine

25 sediments from the Arctic Mid-Ocean Ridge ${ }^{12}$, we detected several Chlamydiae-related

26 contiguous sequences (contigs). This finding prompted us to systematically screen marine

27 sediment cores from a region surrounding Loki's Castle hydrothermal vent field (Fig. 1a). We

28 extracted DNA from 69 sediment samples (Supplementary Table 1) from various core depths,

29 followed by screening using Chlamydiae-specific 16S rRNA gene primers. Chlamydiae were

30 identified in $51(74 \%)$ of the samples ranging in depth from 0.1 to 9.4 meters below seafloor

31 (mbsf). We investigated the chlamydial relative abundance and diversity in 30 of these samples

32 using bacterial-specific 16S rRNA gene amplicon sequencing, resulting in the identification of

33252 operational taxonomic units (OTUs; clustered at 97\% identity; Supplementary Data 1) that

34 could be reliably assigned to Chlamydiae. This analysis revealed notable differences in

35 chlamydiae relative abundance and diversity between samples (Supplementary Data 2), with

36 individual samples showing relative abundances of up to $43 \%$ of the total bacterial community

37 and up to 163 OTUs (Fig. 1b). Furthermore, we found that 155 of the 252 chlamydiae OTUs

38 from our samples (hereafter referred to as "marine sediment chlamydiae") could be identified in

39 at least two samples. We further investigated the diversity of these marine sediment chlamydiae

40 by performing a phylogenetic analysis in which the presently discovered chlamydial OTUs were

41 placed amidst a recently compiled dataset of diverse chlamydiae 16S rRNA gene sequences ${ }^{6}$.

42 This analysis revealed that the overall diversity of marine sediment chlamydiae spanned, and

43 expanded, the known chlamydial diversity (Fig. 1c), revealing several deeply branching clades.

44 To obtain genomic information from these marine sediment chlamydiae, we employed a

45 genome-resolved metagenomics approach (Supplementary Fig. 1) in which we generated 249.6

46 gigabase pairs (Gbp) of paired-end reads from four sediment samples with a high relative

47 abundance and diversity of chlamydial OTUs (Supplementary Fig. 2). Sequence assembly 
generated $5.85 \mathrm{Gbp}$ of contigs larger than 1 kilobase pair (kbp). To assess the diversity of

49 Chlamydiae-related sequences in these metagenome assemblies, we performed phylogenetic

50 analyses of contigs containing 16S rRNA gene sequences or at least five genes of a conserved 15-

51 ribosomal protein gene cluster. These analyses revealed numerous Chlamydiae-related contigs

52 (Supplementary Fig. 2), most of which represented novel lineages that were distantly related to

53 known chlamydiae. Contigs were binned into 24 highly complete (median 95\% completeness,

54 Supplementary Table 2) metagenome-assembled genomes (MAGs) on the basis of their

55 tetranucleotide frequencies and patterns of sequence coverage across samples. They differed

56 markedly in predicted genome size (1.33-1.99 Mbp), GC-content (26.4-48.9\%) and gene content

57 (Fig. 2, Supplementary Figs. 3 and 4).

To robustly infer the evolutionary relationships of the marine sediment chlamydiae to

59 known lineages (Supplementary Table 3), we performed phylogenomic analyses of concatenated

60 conserved marker protein sequence datasets. These analyses, which were designed to minimize

61 potential long branch attraction and compositional bias artefacts, revealed that the newly

62 reconstructed genomes form five new clades of high taxonomic rank (referred to as Chlamydiae

63 Clades (CC) I-IV and Anoxychlamydiales; Fig. 2, Supplementary Fig. 3, Supplementary

64 Discussion). While these phylogenomic analyses form clades that are in agreement with results

65 based on previously available chlamydial genome data ${ }^{13}$, the branching order of these clades

66 inferred differs considerably (Supplementary Discussion). Most marine sediment chlamydiae

67 were placed into one of two well-supported, deeply branching clades, CC-II and

68 Anoxychlamydiales, which also include four MAGs associated with estuary sediment, aquifer

69 groundwater and a drinking water treatment plant ${ }^{14-16}$ (Fig. 2). Anoxychlamydiales are unique

70 among Chlamydiae as they comprise members with gene repertoires indicative for an anaerobic

71 lifestyle and will be treated in more detail in a complementary study (manuscript in prep.). 
72 Together, CC-I, CC-II, CC-III and Anoxychlamydiales form a superclade that is mainly 73 comprised of uncultivated members represented by MAGs (Fig. 2, Supplementary Fig. 3). While

74 the lifestyles of most members of this superclade remain elusive, the distinctive gene repertoires

75 of the various clades point at functional differences (Supplementary Figs. 3 and 4). A second

76 chlamydial superclade is comprised of the environmental chlamydiae, CC-IV and Chlamydiaceae

77 (Fig. 2, Supplementary Fig. 3) and includes five of the marine sediment chlamydiae MAGs.

78 While two of these MAGs affiliate with the environmental chlamydiae, which comprise

79 symbionts of single-celled eukaryotes such as amoebozoa ${ }^{7}$, three MAGs resolve into a previously

80 unknown clade referred to as CC-IV. Previous studies have suggested that Chlamydiaceae, a

81 family that includes important animal pathogens such as the human pathogen Chlamydia

82 trachomatis $^{17}$, represents a deep-branching clade of the Chlamydiae phylum ${ }^{13}$. However, our

83 phylogenetic analyses strongly support that Chlamydiaceae and CC-IV represent sisterclades,

84 which together share a common ancestor with environmental chlamydiae (Fig. 2). Hence our

85 results indicate that Chlamydiaceae represent a clade that was formed relatively late in

86 chlamydiae evolution (Fig. 2), and that features specifically associated with pathogenicity in the

87 Chlamydiaceae evolved much more recently than previously assumed.

88 Uncovering the sister relationship of CC-IV and the pathogenic Chlamydiaceae allowed

89 us to re-evaluate the evolutionary events leading to their emergence (Fig. 3a). The genome sizes

90 of CC-IV MAGs, while generally smaller than those of environmental chlamydia (Fig 2.), are

91 larger than those of Chlamydiaceae (Fig. 2), suggesting that the latter have been subjected to

92 genome reduction, a feature often observed in animal pathogens ${ }^{18}$. However, when considering

93 only the gene set conserved across environmental chlamydiae, these differences are less drastic

94 (Fig. 3b), and distribution patterns of Clusters of Orthologous Group (COG) categories found in

95 CC-IV lineages more closely resemble that of environmental chlamydiae than Chlamydiaceae 
96 (Supplementary Fig. 5). Since their divergence from CC-IV, Chlamydiaceae have experienced

97 reductive genome evolution (Fig. 3a-b, Supplementary Fig. 5), including the loss of several core

98 components of central carbon metabolism, and de novo biosynthesis of nucleotides and amino

99 acids (Supplementary Fig. 4, Supplementary Discussion). At the same time, they have acquired a

100 small number of genes primarily linked to host-interaction and virulence, in addition to a notable

101 set of conserved genes with unknown functions (Supplementary Fig. 6, Supplementary

102 Discussion). It is likely that some of these unique features of the Chlamydiaceae are linked to the

103 emergence of host-specificity. Further comparative analyses of CC-IV and Chlamydiaceae

104 genomes revealed that only seven gene families are uniquely shared between these clades

105 (Supplementary Fig. 7, Supplementary Discussion). This gene set is highly conserved across the

106 Chlamydiaceae family, hinting at their putative importance in their adaptation to a pathogenic

107 lifestyle. In addition, phylogenetic analyses of gene families with multiple homologs reveal gene

108 duplication events have occurred both before and after the divergence of the Chlamydiaceae from

109 CC-IV (Supplementary Fig. 7, Supplementary Discussion). However, the exact functions of these

110 genes in Chlamydiaceae are currently unknown and our analyses suggest that they should

111 represent a priority for future functional investigations. CC-IV genomes also encode homologs of

112 several flagellar proteins (Supplementary Discussion), which clustered with flagellar components

113 recently identified within distantly-related chlamydiae sampled from marine waters ${ }^{19}$

114 (Supplementary Fig. 8), indicating motility as a sharp difference between CC-IV and

115 Chlamydiaceae.

116 All previously known members of the Chlamydiae, including environmental lineages, are

117 obligate intracellular symbionts of eukaryotic hosts ${ }^{20}$ and display an obligate host-association for

118 replication ${ }^{10}$. Similar to previously characterized lineages ${ }^{20}$, the herein identified marine sediment

119 chlamydiae encode various homologs of proteins associated with this typical chlamydial lifestyle 
120 (Supplementary Fig. 4, Supplementary Discussion). For example, the marine sediment

121 chlamydiae contain NF-T3SS (Supplementary Figs. 8 and 9) and NF-T3SS-specific effectors

122 (Supplementary Data 3, Supplementary Discussion). NF-T3SS components are typically present

123 in obligate bacterial symbionts that have a eukaryotic host, but they are also observed in free-

124 living lineages (Supplementary Discussion). Similarly, most of these genomes contain genes

125 encoding other secretion systems (Supplementary Fig. 10 and 11, Supplementary Discussion),

126 some of which have been described to participate in adhesion and invasion in Chlamydiaceae.

127 However, these systems have been shown to have alternative functions in other bacteria

128 (Supplementary Discussion). Nucleotide transporters (NTTs), and in particular ATP/ADP

129 transporters, are a characteristic feature of Chlamydiae and other bacteria typically associated

130 with eukaryotic hosts ${ }^{21}$. All marine sediment chlamydiae genomes encode multiple NTT

131 homologs (Supplementary Figs. 4 and 12, Supplementary Discussion), including possible

132 ATP/ADP NTTs, which cluster together with other chlamydial sequences in phylogenetic

133 analyses (Supplementary Fig. 12). Pathways for the de novo biosynthesis of nucleotides and

134 amino acids are often incomplete in obligate intracellular symbionts ${ }^{8,18}$. Indeed, despite

135 identifying chlamydial lineages with the genetic capacity to synthesize both purine and

136 pyrimidine nucleotides de novo, we did not observe lineages capable of de novo synthesis of all

137 nucleotides and amino acids (Supplementary Fig. 4, Supplementary Discussion).

138 Since the genomes of these marine sediment chlamydiae encode the typical features of

139 host-dependency found in previously characterized chlamydiae ${ }^{9}$, we expected to find indications

140 of potential eukaryotic hosts in the marine sediment samples. While attempts to amplify $18 \mathrm{~S}$

141 rRNA genes from DNA isolated from these sediment samples were unsuccessful, screening the

142 four marine sediment metagenome datasets resulted in the identification of a few 18S rRNA gene

143 fragments (Supplementary Table 4, Supplementary Discussion). However, no such sequences 
144 could be identified in the metagenome of the marine sediment sample with the highest

145 Chlamydiae relative abundance (Fig. 1b). Given the absence of obvious host candidates, we

146 explored the possibility that these genome sequences derive from persistent marine sediment

147 chlamydiae cells, such as from elementary bodies, which can survive outside of host cells and

148 even remain metabolically active, despite being unable to replicate ${ }^{22,23}$. Estimation of replication

149 rates based on sequence read-coverage ${ }^{24}$ indicated that these MAGs are derived from actively

150 dividing marine sediment chlamydiae (Fig. 4b). Thus, our results suggest that at least some of

151 these marine sediment chlamydiae are not associated with eukaryotic hosts.

152 Finally, we investigated chlamydial diversity in environments other than marine

153 sediments. An analysis of publicly available 16S rRNA gene amplicon datasets from a variety of

154 environments revealed that fresh water, ground water, salt marshes and wastewater often harbour

155 diverse and abundant chlamydiae (Fig. 4b). These findings likely represent considerable

156 underestimations of chlamydial diversity and relative abundance since mismatches in commonly

157 used 16S rRNA primer sets (e.g., Earth Microbiome Project ${ }^{25}$, Supplementary Table 5) generally

158 do not capture known Chlamydiae diversity (Supplementary Discussion).

159 Our work reports the existence and genomic characterization of an extended diversity of

160 Chlamydiae-related lineages in deep marine sediments and provides insights into the evolution

161 and diversification of the Chlamydiae phylum. Using sophisticated phylogenomic methods, we

162 used a robust phylogenomic framework for investigating Chlamydiae evolution. Furthermore, we

163 identified several new Chlamydiae clades of high taxonomic rank, including a sister clade of the

164 pathogenic Chlamydiaceae, which provided insights into the early evolution of this family.

165 Altogether, our findings indicate that Chlamydial diversity and abundance has been

166 underappreciated in environmental surveys, and our observations represent a shift in our view of

167 the environmental distribution of Chlamydiae. These results indicate differences in basic ecology 
169 evolution, including the emergence of host dependency and pathogenicity.

\section{References}

1731 Bachmann, N. L., Polkinghorne, A. \& Timms, P. Chlamydia genomics: providing novel insights into chlamydial biology. Trends Microbiol 22, 464-472 (2014).

2 Nunes, A. \& Gomes, J. P. Evolution, phylogeny, and molecular epidemiology of Chlamydia. Infect Genet Evol 23, 49-64 (2014).

3 Elwell, C., Mirrashidi, K. \& Engel, J. Chlamydia cell biology and pathogenesis. Nat Rev Microbiol 14, 385-400 (2016).

4 Amann, R. et al. Obligate Intracellular Bacterial Parasites of Acanthamoebae Related to Chlamydia spp. Applied and Environmental Microbiology 63, 115-121 (1997).

5 Horn, M. \& Wagner, M. Evidence for additional genus-level diversity of Chlamydiales in the environment. FEMS Microbiology Letters 204, 71-74 (2001).

6 Lagkouvardos, I. et al. Integrating metagenomic and amplicon databases to resolve the phylogenetic and ecological diversity of the Chlamydiae. ISME J 8, 115-125 (2014).

7 Horn, M. Chlamydiae as symbionts in eukaryotes. Annu Rev Microbiol 62, 113-131, doi:10.1146/annurev.micro.62.081307.162818 (2008).

8 Horn, M. et al. Illuminating the evolutionary history of chlamydiae. Science 304, 728730, doi:10.1126/science.1096330 (2004).

9 Omsland, A., Sixt, B. S., Horn, M. \& Hackstadt, T. Chlamydial metabolism revisited: interspecies metabolic variability and developmental stage-specific physiologic activities. FEMS Microbiol Rev 38, 779-801, doi:10.1111/1574-6976.12059 (2014).

10 Taylor-Brown, A., Vaughan, L., Greub, G., Timms, P. \& Polkinghorne, A. Twenty years of research into Chlamydia-like organisms: a revolution in our understanding of the biology and pathogenicity of members of the phylum Chlamydiae. Pathog Dis 73, 1-15 (2015).

11 Pedersen, R. B. et al. Discovery of a black smoker vent field and vent fauna at the Arctic Mid-Ocean Ridge. Nat Commun 1, doi:10.1038/ncomms1124 (2010).

12 Spang, A. et al. Complex archaea that bridge the gap between prokaryotes and eukaryotes. Nature 521, 173-179, doi:10.1038/nature14447 (2015).

13 Pillonel, T., Bertelli, C. \& Greub, G. Environmental Metagenomic Assemblies Reveal Seven New Highly Divergent Chlamydial Lineages and Hallmarks of a Conserved Intracellular Lifestyle. Front Microbiol 9, 79, doi:10.3389/fmicb.2018.00079 (2018).

14 Baker, B. J., Lazar, C. S., Teske, A. P. \& Dick, G. J. Genomic resolution of linkages in carbon, nitrogen, and sulfur cycling among widespread estuary sediment bacteria. Microbiome 3, 14, doi:10.1186/s40168-015-0077-6 (2015).

15 Anantharaman, K. et al. Thousands of microbial genomes shed light on interconnected biogeochemical processes in an aquifer system. Nat Commun 7, 13219, 208 doi:10.1038/ncomms13219 (2016). 
Pinto, A. J. et al. Metagenomic Evidence for the Presence of Comammox Nitrospira-Like Bacteria in a Drinking Water System. mSphere 1, doi:10.1128/mSphere.00054-15 (2016).

17 Stephens, R. S. et al. Genome Sequence of an Obligate Intracellular Pathogen of Humans: Chlamydia trachomatis. Science 282, 754-759 (1998).

18 Toft, C. \& Andersson, S. G. E. Evolutionary microbial genomics: insights into bacterial host adaptation. Nature Reviews Genetics 11, 465-475, doi:10.1038/nrg2798 (2010).

19 Collingro, A. et al. Unexpected genomic features in widespread intracellular bacteria: evidence for motility of marine chlamydiae. ISME $J$ 11, 2334-2344, doi:10.1038/ismej.2017.95 (2017).

20 Collingro, A. et al. Unity in variety--the pan-genome of the Chlamydiae. Mol Biol Evol 28, 3253-3270 (2011).

21 Schmitz-Esser, S. et al. ATP/ADP Translocases: a Common Feature of Obligate Intracellular Amoebal Symbionts Related to Chlamydiae and Rickettsiae. Journal of Bacteriology 186, 683-691, doi:10.1128/jb.186.3.683-691.2004 (2004). Haider, S. et al. Raman microspectroscopy reveals long-term extracellular activity of Chlamydiae. Mol Microbiol 77, 687-700, doi:10.1111/j.1365-2958.2010.07241.x (2010). link between activity and infectivity in Chlamydiae. PLoS Pathog 9, e1003553, doi:10.1371/journal.ppat.1003553 (2013).

Acknowledgements We thank M. Horn, L. Guy, S. Abby, L. Juzokaite, A. E. Lind, K. Zaremba-

Niedzwiedzka and E. C. Fernandez for technical assistance and/or for useful advice and

party and the entire crew on board the Norwegian research vessel G.O. Sars during the summer Infrastructure sequencing platforms at the Science for Life Laboratory at Uppsala University, a national infrastructure supported by the Swedish Research Council (VR-RFI) and the Knut and Alice Wallenberg Foundation. We thank the Uppsala Multidisciplinary Center for Advanced

243 Computational Science (UPPMAX) at Uppsala University and the Swedish National

244 Infrastructure for Computing (SNIC) at the PDC Center for High-Performance Computing for 
245 providing computational resources. This work was supported by grants of the European Research

246 Council (ERC Starting grant 310039-PUZZLE_CELL), the Swedish Foundation for Strategic

247 Research (SSF-FFL5) and the Swedish Research Council (VR grant 2015-04959) to T.J.G.E.

248 C.W.S. is supported by a European Molecular Biology Organization long-term fellowship

249 (ALTF-997-2015) and the Natural Sciences and Engineering Research Council of Canada

250 postdoctoral research fellowship (PDF-487174-2016). Funding was received from the European

251 Union's Horizon 2020 research and innovation program under the respective Marie Sklodowska-

252 Curie grant agreements 625521 (to A.S.) and 704263 (to L.E.). A.S. is supported by the Swedish

253 Research Council (VR starting grant 2016-03559) and the NWO-I foundation of the Netherlands

254 Organisation for Scientific Research (WISE fellowship).

256 Author Contributions T.J.G.E. and A.S. conceived the study, and J.E.D. and T.J.G.E.

257 performed the experimental design. S.L.J. provided environmental samples. S.L.J. and J.E.D.

258 performed DNA extractions and performed PCR-based screening. J.E.D. generated 16S rRNA

259 gene amplicons. F.H. and J.E.D. performed metagenomic sequence assemblies. J.E.D., J.M. and

260 F.H. performed genome-resolved metagenomics analyses. J.E.D., F.H. and T.J.G.E. analyzed 16S

261 rRNA gene amplicon sequence data. J.E.D., C.S., D.T., L.E., J.M., and A.S. analyzed genomic

262 data and performed phylogenetic analyses. J.E.D., C.S., L.E., D.T., A.S. and T.J.G.E. interpreted

263 the obtained data and results. T.J.G.E. and J.E.D wrote, and all authors edited and approved, the

264 manuscript. 
a

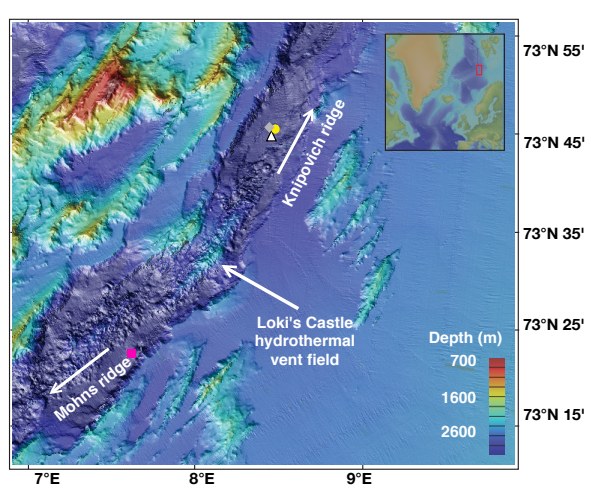

b

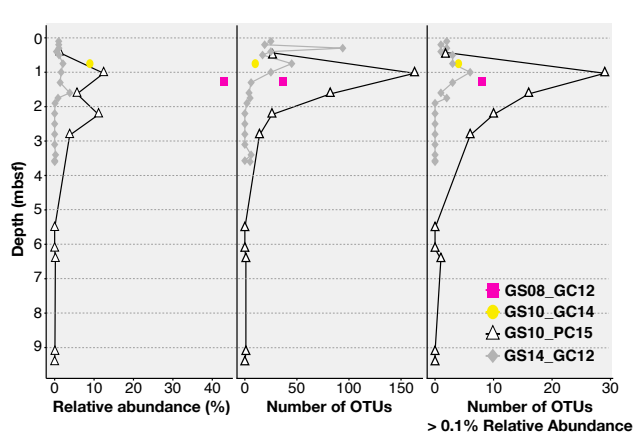

c

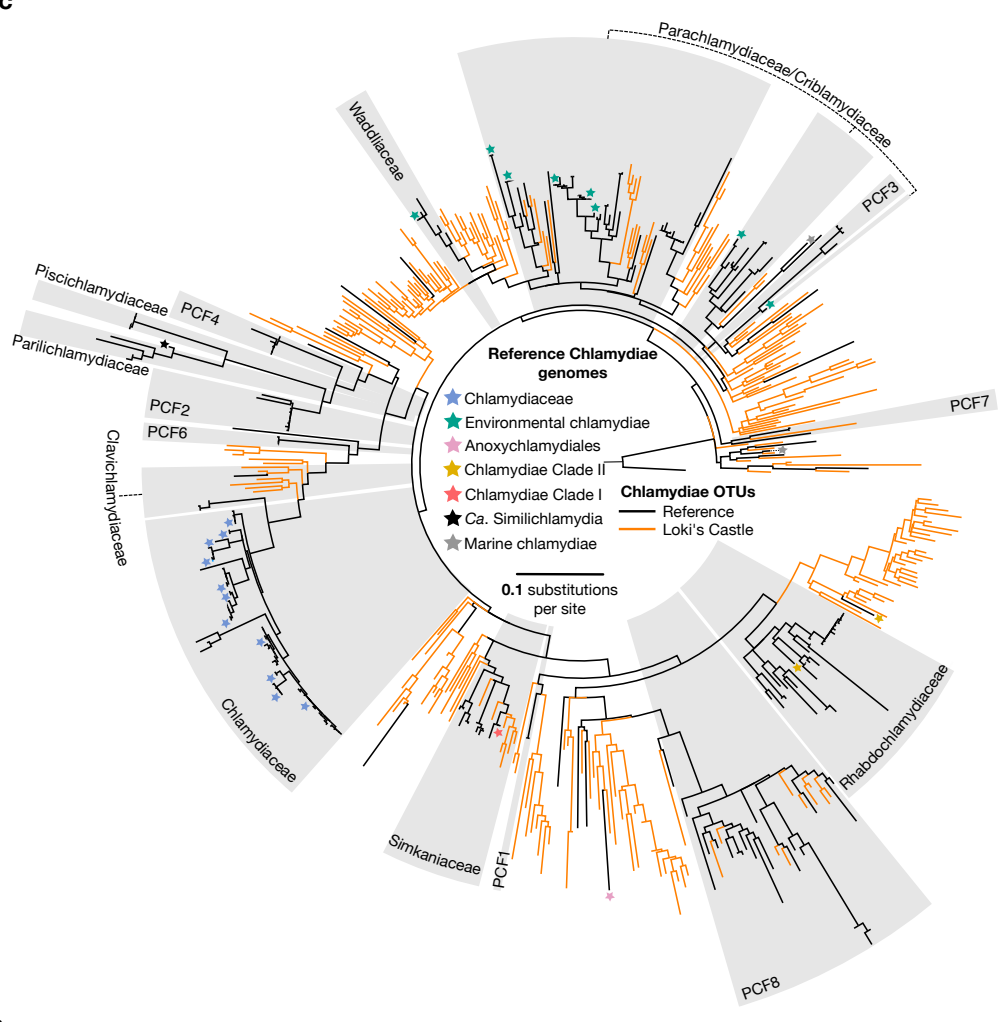

Figure 1. Chlamydiae are diverse and abundant in Loki's Castle marine sediments. a, Bathymetric map of sediment core sampling locations taken northeast (GS10_GC14, GS10_PC15 and GS14_GC12) and southwest (GS08_GC12) of Loki's Castle hydrothermal vent field. $\mathbf{b}$, Chlamydial relative abundance, OTU number and abundant OTUs, as a factor of depth (meters below seafloor, mbsf) based on bacterial 16S rRNA gene amplicons. c, Maximumlikelihood (ML) phylogenetic tree (520 taxa, 476 sites) of marine sediment chlamydiae 16S rRNA gene sequences from amplicon OTUs (orange) and a reference (black) dataset, rooted using PVC taxa as an outgroup, inferred using IQ-TREE with the GTR+R7 model of evolution. Previously sequenced chlamydial genomes and metagenomic bins are labelled with stars. 

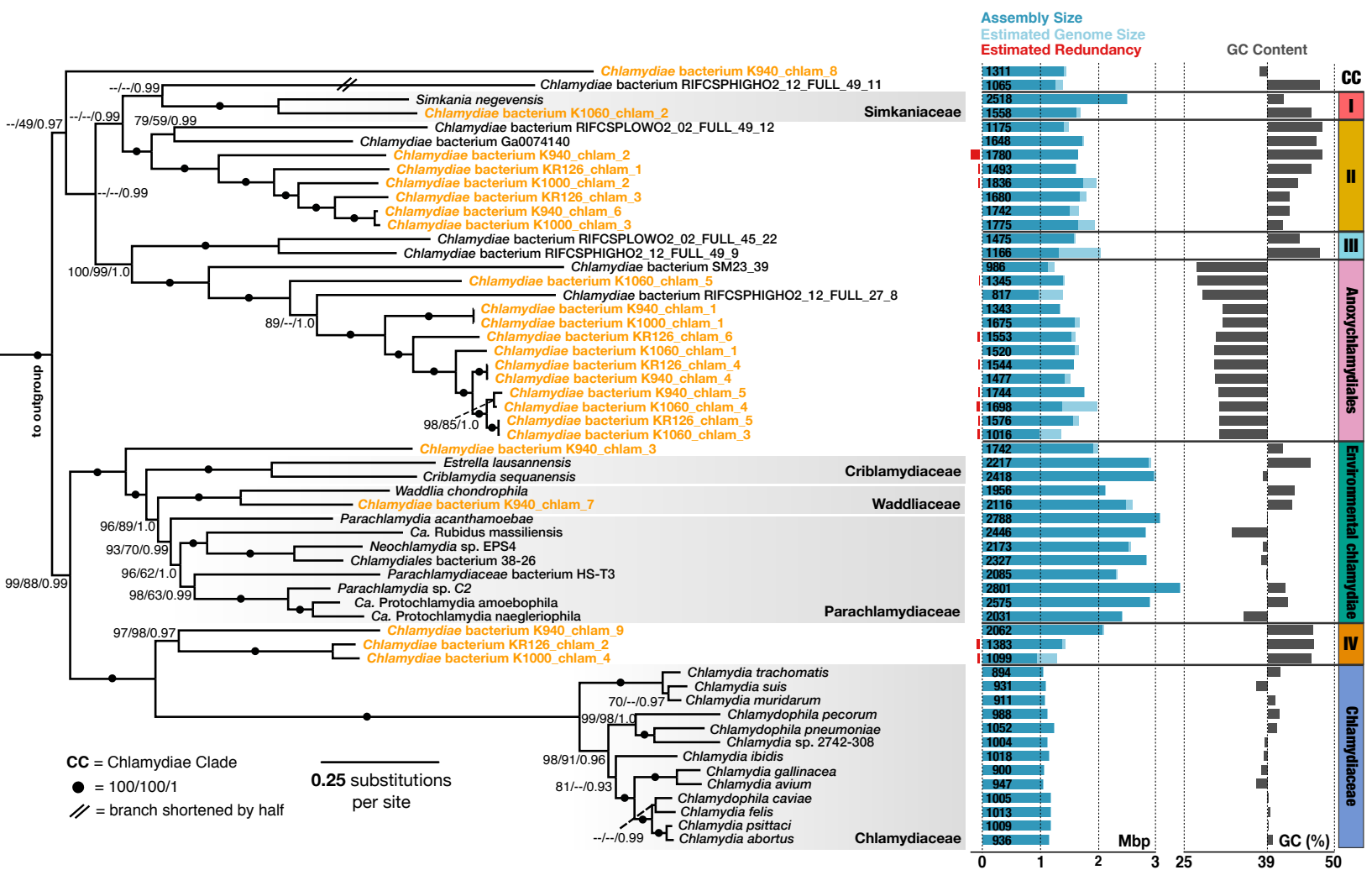

Figure 2. Marine sediment lineages span Chlamydiae species phylogeny. Bayesian phylogenetic tree of 38 concatenated single-copy conserved marker proteins in which marine sediment chlamydiae and previously characterized chlamydial representatives are shown in orange and black fonts, respectively. Previously established chlamydial families are shaded in grey. Branch support values were mapped onto the tree in the following order: non-parametric bootstrap support values (BV) for the full alignment (8006 sites) and reduced alignment (6005 sites after removal of the top $25 \%$ compositionally heterogeneous sites), each under the $\mathrm{LG}+\mathrm{C} 60+\mathrm{G}+\mathrm{F}$ derived PMSF approximation estimated by IQ-TREE, and posterior probability (PP) support values under the $\mathrm{CAT}+\mathrm{GTR}+\Gamma 4$ model of evolution inferred with Phylobayes. For each species, the genome bin size, estimated genome size and redundancy are reported in Mbp along with the number of predicted open reading frames, and GC content. 
a

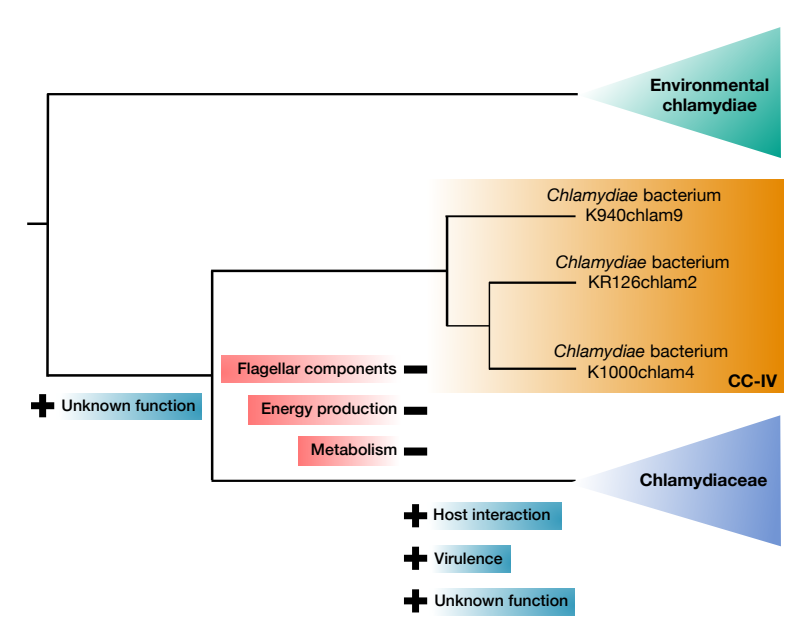

b

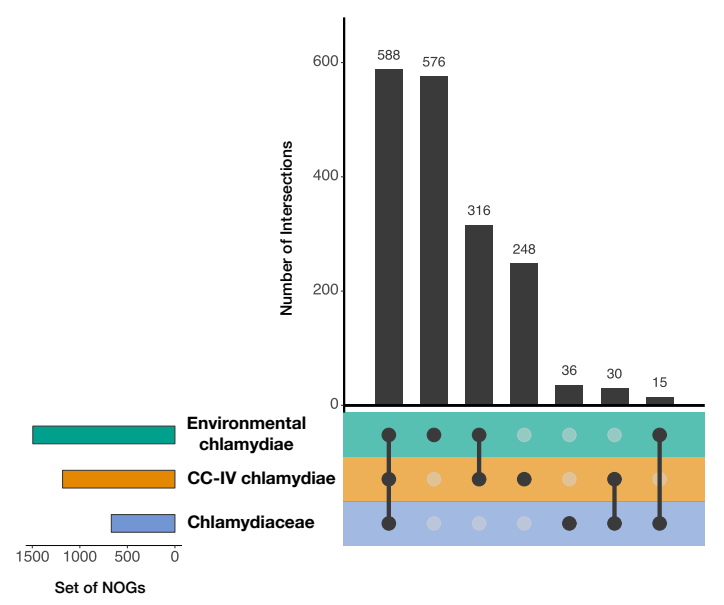

Figure 3. Gene content evolution in Chlamydiaceae. a, Schematic overview of lost and acquired cellular features, based on presence and absence patterns of NOGs (Supplementary Data 4), in pathogenic Chlamydiaceae, inferred from sister clade CC-IV. b, Plot showing intersections of NOGs conserved (in a third of lineages affiliated with each clade, Supplementary Table 2 and 3 ) across environmental chlamydiae, CC-IV and Chlamydiaceae. 
a

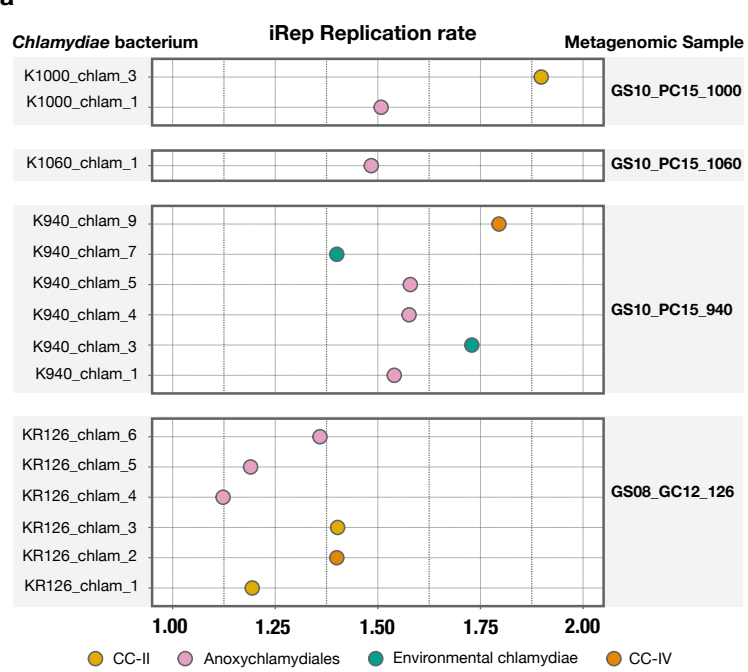

b

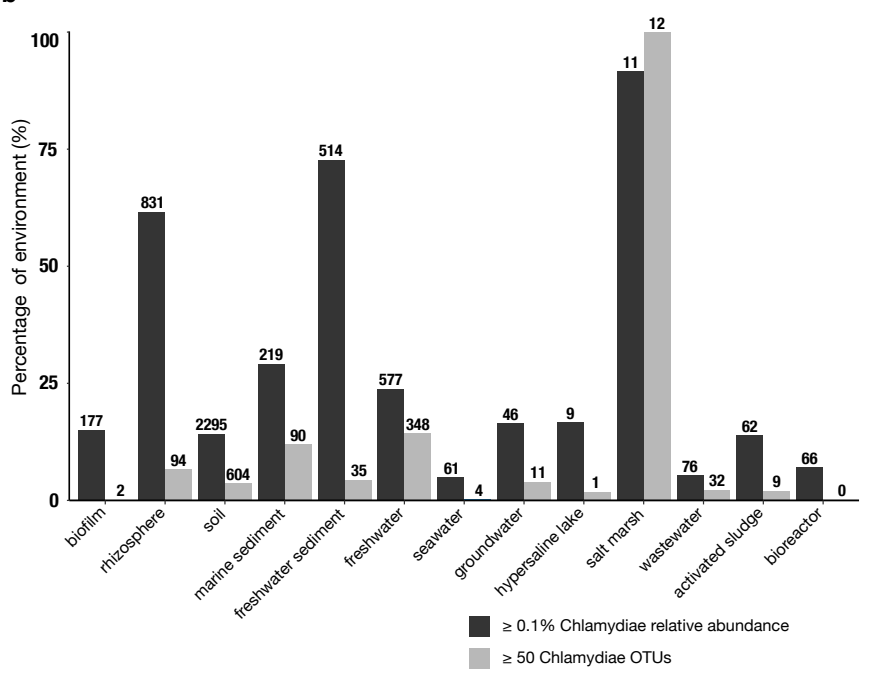

Figure 4. Estimated iRep replication rate of marine sediment chlamydiae, and bar plot showing presence of chlamydiae in diverse environments. a, Replication rates of marine sediment chlamydiae inferred using iRep. Indicates the proportion of the microbial population represented by the metagenome-assembled genome that is actively dividing. b. Percentage of samples from selected environments which contain a chlamydial relative abundance of $\geq 0.1 \%$, or $\geq 50$ OTUs based on publicly available $16 \mathrm{~S}$ rRNA gene amplicon datasets. Values above each bar represent the absolute number of samples. 


\section{Methods}

\section{Sample acquisition}

376 Sediment cores were retrieved from the Arctic Mid-Ocean Ridge near Loki's Castle ${ }^{11}$

377 hydrothermal vent field over multiple sampling expeditions: GS08_GC12 in $2008(3.3 \mathrm{~m})^{26}$,

378 GS10_GC14 (2 m) and GS10_PC15 (11.2 m) in 2010²7, and GS14_GC12 (3.6 m) in 2014.

379 Sediment samples at various depths (Supplementary Table 1) were collected on-board and 380 immediately frozen for prospective microbiological analysis. Geochemistry and sediment

381 characteristics of these samples have been published previously ${ }^{26,27}$, except in the case of

382 GS14_GC12.

\section{Sample screening and bacterial 16S rRNA gene amplicon sequencing}

384 DNA was extracted using the PowerLyzer ${ }^{\circledR}$ PowerSoil ${ }^{\circledR}$ DNA kit in conjunction with the

385 PowerLyser® 24 homogenizer as per manufacturer's instructions (MOBIO) with minor

386 modifications using $0.5-0.7 \mathrm{~g}$ of sediment and the addition of polyadenosine to increase DNA

387 yield $^{28}$. Taxonomic coverage of all primer pairs, for both sample screening and amplicon

388 sequencing, was tested in silico using SILVA TestPrime ${ }^{29}$ with the SSU r132 RefNR database

389 (Supplementary Table 5). All primers used have been published previously ${ }^{29,30}$ except for Chla-

390 310-a-20 which was designed with PRIMROSE ${ }^{31}$ (Supplementary Table 5). Reaction conditions

391 for each primer pair are found in Supplementary Table 5. Primer pair Chla-310-a-20 and S-*_

392 Univ-1100-a-A-15 ${ }^{29}$, which amplifies an approximately 800 bp region of the $16 \mathrm{~S}$ rRNA gene,

393 was used to screen 69 Loki's Castle marine sediment samples (Supplementary Table 1) for

394 Chlamydiae. Primer pair $574 * \mathrm{f}^{30}$ and $1132^{30}$ were used to screen for $18 \mathrm{~S}$ rRNA genes in these

395 same sediment samples. The latter primer pair did not yield positive amplification products, in 
396 spite of its broad taxonomic coverage of known eukaryotic 18S rRNA genes (Supplementary

397 Table 5).

Thirty sediment samples with positive PCR screening results for Chlamydiae were selected for further investigation. Bacterial-specific 16S rRNA gene amplicons were sequenced 400 using a two-step PCR approach. Primer sequences and reaction conditions are reported in

401 Supplementary Table 5. In the first step, a ca. 500 bp region of the 16S rRNA gene was amplified 402 in triplicate, to account for random PCR drift ${ }^{32}$, using bacterial primers (S-D-0564-a-S-15 and S403 D-Bact-1061-a-A-17 ${ }^{29}$ ). For each reaction, replicates were pooled and purified using magnetic

404 AMPure XP beads (Agencourt). In the second PCR step, libraries were constructed using adaptor 405 sequences and reaction conditions from the TruSeq DNA LT Sample Prep Kit (Illumina), before 406 sequencing with Illumina MiSeq $\left(2 \times 300\right.$ bp). Using cutadapt ${ }^{33}$ v. 1.10 sequence reads shorter 407 than 100 bases were filtered out, 3' ends trimmed to a minimum Phred quality score of 10, and 408 primer sequences removed. Forward and reverse reads were merged using VSEARCH ${ }^{34}$ v. 1.11 .1 409 (--fastq-minovlen 16), de-replicated (--derep_fulllength), and clustered into centroid OTUs 410 (threshold $=97 \%$ ). Chimeras were detected and removed using UCHIME ${ }^{35}$ with the

411 SILVA123.1_SSUref_tax:99 database ${ }^{36}$. Taxonomy was assigned using the LCAClassifier ${ }^{37}$ from

412 CREST-2.0.5 with silvamod106 as the reference database.

\section{Metagenome sequencing and assembly}

414 The Fast DNA Max Spin kit and Fast DNA Spin kit (for GS10_PC15_940 only, 4 replicates 415 pooled) were used according to manufacturer's protocols (MP Biomedical), with the addition of 416 polyadenosine, to extract DNA from sediment samples chosen for further analysis 417 (GS08_GC12_126, GS10_PC15_940, GS10_PC15_1000, and GS10_PC15_1060). Libraries 418 were prepared using the Nextera DNA Library Prep kit (Illumina) with 25 ng of input DNA, and 419 sequenced with Illumina HiSeq in rapid-mode (2x 250 bp). For GS10_PC15_1060, reads from 
420 three separate HiSeq runs and undetermined reads (reads with barcode mismatches) from one run

421 were combined. Quality control to remove low-quality reads was performed using

422 Trimmomatic ${ }^{38} 0.35$ with the options: SLIDINGWINDOW:4:12, MINLEN:50,

423 ILLUMINACLIP:TruSeq Illumina Universal Adaptors. FastQC ${ }^{39}$ v.011.4 was used to visually

424 evaluate sequence quality before and after processing. Using the fq2fa program from the IDBA-

$425 \mathrm{UD}^{46}$ package, paired reads were interlaced and Ns removed, using the options 'merge' and

426 'filter' (except for GS10_PC15_1060 reads, where Ns were retained, as it improved the

427 assembly). Trimmed reads from each sample were subjected to iterative de novo assembly using

428 IDBA-UD 1.0 .9 (minimum $\mathrm{k}$-mer size $=20$ and maximum $\mathrm{k}$-mer size $=124$, except for

429 GS08_GC12_126 for which maximum k-mer size = 100). Assembly quality and statistics

430 (Supplementary Fig. 2) were assessed using QUAST $^{40}$ v3. Open reading frames (ORFs) across

431 assembled contigs in each metagenome were called with Prodigal $^{41}$ v.2.6.3.

\section{Assesment of metagenome microbial composition}

433 To investigate the microbial composition of the samples, 'ribocontigs', i.e. a contig encoding at

434 least 5 of 15 ribosomal proteins found in an operon conserved across prokaryotes ${ }^{42}$, were

435 identified in the metagenomic assemblies using the RP15 pipeline ${ }^{43}$. As part of this, a maximum-

436 likelihood (ML) phylogeny using the $\mathrm{LG}+\mathrm{C} 60+\mathrm{G}$ model of evolution (Supplementary Fig. 2) was

437 inferred from a dataset consisting of concatenated ribosomal proteins extracted from

438 metagenomic ribocontigs and 90 phylogenetically diverse reference taxa including bacteria and

439 archaea $^{44}$ in addition to PVC representative species (Supplementary Tables 3 and 6).

440 Obtaining Chlamydiae metagenome assembled genomes

441 A differential coverage binning approach was used to obtain metagenome-assembled genomes

442 (MAGs). For each metagenome assembly, contig coverage was estimated using pseudoalignment

443 with Kallisto ${ }^{45} 0.42 .5$, with sequence reads from each of the four samples. Differential coverage 
444 profiles were generated for each assembly using a freely available script

445 (github.com/EnvGen/toolbox/tree/master/scripts/kallisto_concoct/input_table.py), provided by

446 Johannes Alneberg. To give more statistical weight to longer contigs and to reduce the impact of

447 chimeric sequences ${ }^{46}$, contigs larger than $20 \mathrm{~kb}$ were split into $10 \mathrm{~kb}$ fragments. CONCOCT $^{46}$

448 v.0.40 was used to cluster contigs within each focal assembly into MAGs, using their differential

449 coverage profile ${ }^{47}$, tetranucleotide frequency, and different contig length cut-offs $(1 \mathrm{~kb}, 2 \mathrm{~kb}$ and

$4503 \mathrm{~kb}$ ). Due to the large diversity in sample GS10_PC15_1060, the maximum number of bins was

451 adjusted (1500 bins for $1 \mathrm{~kb}$, and 1000 bins for 2 and $3 \mathrm{~kb}$ length cut-offs). Putative chlamydial

452 metagenomic bins were identified by phylogenomic analyses of concatenated ribosomal proteins

453 encoded on ribocontigs (Supplementary Fig. 2). Completeness and redundancy was assessed

454 using the micomplete ${ }^{48}$ pipeline (without weighting), provided by Lionel Guy

455 (https://bitbucket.org/evolegiolab/micomplete) using a custom marker set (Supplementary Table

456 7) corresponding to genes present in complete Chlamydiae genomes (Supplementary Table 3 ).

457 For each ribocontig, the corresponding metagenomic bin with the highest completeness and

458 lowest redundancy across the CONCOCT iterations was selected for further analysis. Chlamydial

459 metagenome bins were subjected to manual cleaning using mmgenome ${ }^{49}$, resulting in medium

460 and high quality MAGs (Supplementary Table 2) based on MIMAG standards ${ }^{56}$. Differential

461 coverage across samples, GC content, linkage, the presence of chlamydial-specific marker

462 proteins $^{20}$, and single-copy bacterial marker proteins (Supplementary Table 7) were visualized

463 using mmgenome ${ }^{50}$. Contigs with profiles diverging from the majority of contigs were removed.

464 Linkage information, i.e. information about which contigs are connected by read pairs, was

465 calculated with read mapping using Bowtie $2^{51}$, followed by application of the script

466 bam_to_linkage.py from $\mathrm{CONCOCT}^{46}$. After generating the final MAGs, contigs that had been

467 split into $10 \mathrm{~kb}$ contig fragments were joined again if more than half of the fragments of a 
468 specific contig were assigned to a specific MAG. Otherwise, all fragments of the contig in

469 question were discarded.

470 Selection of published PVC genomes for comparative and phylogenetic analysis

471 Selection of PVC superphylum representatives was facilitated by a phylogenetic analysis of

472 ribocontigs from PVC member genomes available in NCBI (as of February $6^{\text {th }}, 2017$ ). Using the

473 RP15 pipeline ${ }^{43}$, a maximum-likelihood (ML) phylogeny of these ribocontigs was inferred, using

474 RAxML ${ }^{52}$ 8.2.4 under the PROTCATLG model of evolution. Branch support was estimated

475 through 100 rapid bootstrap replicates ('-f a') (Supplementary Fig. 13). Phylogenetically diverse

476 representatives from non-Chlamydiae PVC phyla were selected (Supplementary Table 6) to be

477 used as an outgroup for phylogenomic analyses. With the exception of Chlamydiaceae (for which

478 we used genomes classified as reference or representative in NCBI), all Chlamydiae genomes

479 (Supplementary Table 3) were used in protein phylogenies and comparative genomics analyses.

480 For determining interspecies relationships within the Chlamydiae phylum (Supplementary Fig.

481 13), only one representative was kept whenever several chlamydial genomes had a near-identical

482 phylogenetic placement (Supplementary Table 3). Chlamydial species representative MAGs and

483 single-cell assembled genomes (SAGs), which were made available on NCBI subsequently

484 (between February $6^{\text {th }}, 2017$ and April $18^{\text {th }}$, 2018, Supplementary Table 3), were included in

485 comparative genomics and phylogenetic analyses (Supplementary Fig. 4).

\section{Protein clustering and gene annotation}

487 Gene features of marine sediment chlamydiae MAGs (Supplementary Table 2) were annotated

488 with Prokka ${ }^{53}$ v1.12, using a version that allows for partial gene prediction (GitHub pull request 489 \#219). All protein sequences from both chlamydiae in NCBI (Supplementary Table 3) and from

490 those in this study (Supplementary Table 2) were searched against databases as follows: top hits 491 with and excluding Chlamydiae against the $n r$ database and taxonomic classification (Lowest 
492 Common Ancestor (LCA) algorithm, '-f 102') using blastp (--more-sensitive) from DIAMOND ${ }^{54}$

493 aligner v0.9.19.120; PFAM (PF) ${ }^{55}$ and Interpro (IPR) ${ }^{56}$ domains, and MetaCyc ${ }^{57}$ and $\mathrm{KEGG}^{58,59}$

494 pathway annotations, were assigned using Interproscan ${ }^{60}$ version 5.22-61.0; KEGG 'KO'

495 numbers were assigned using GhostKOALA ${ }^{61}$. Protein sequences were also mapped to the

496 eggNOG orthologous groups ${ }^{62}$ version 4.5 using eggNOG-mapper ${ }^{63}$, at both the universal '-d

497 NOG' and bacterial level '-d BACT'. The presence of proteins of interest across Chlamydiae

498 genomes were assessed using these annotations and database searches (Supplementary Data 3).

499 The presence of amino acid and nucleotide de novo biosynthesis and central carbon metabolism

500 pathways (Supplementary Data 3 ) were manually investigated using $\mathrm{KEGG}^{58}$ KO number 501 assignments.

502 Detection of flagellar genes, secretion systems, NF-T3SS secreted proteins, eukaryotic-like

\section{3 domains and subcellular targeting signals in the host}

504 Genes related to secretion systems and the flagellum were detected for most chlamydiae using

505 MacSyFinder ${ }^{71}$ with the protein models built by Abby et al. ${ }^{64}$, using the mode 'gembase' for

506 complete genomes and 'unordered' for incomplete genomes. We predicted NF-T3SS secreted

507 proteins, eukaryotic-like domains (ELD), and putative subcellular targeting signals to eukaryotic

508 cellular compartments for all predicted proteins from chlamydiae (Supplementary Table 2 and 3)

509 and from nine well-characterized PVC representatives with free-living lifestyles. For this, we

510 used EffectiveDB ${ }^{65}$ in 'genome mode', i.e. enabling the prediction of secretion systems and the

511 discovery of novel ELD (Supplementary Data 3).

\section{Phylogenetic analyses}

513 Unless otherwise stated, gene or protein sequences were aligned using MAFFT-L-INS-i ${ }^{74} \mathrm{v}^{7.271}$

514 and trimmed with trimAl ${ }^{66}$ v1.4 (--gappyout). Identical sequences were removed, and alignments

515 were inspected manually. ML phylogenetic analyses were performed using IQ-TREE ${ }^{67} 1.5 .3$ with 
516 automated model selection ${ }^{68}$ among the following models: the empirical LG model ${ }^{69}$ the $^{2}$

517 empirical profile mixture models ( $\mathrm{C} 10$ to $\mathrm{C} 60)$ combined with the LG exchangeability matrix

518 (e.g., $\mathrm{LG}+\mathrm{C} 10)^{70}$, with or without empirically determined amino acid frequencies $(+\mathrm{F})$, and free

519 or gamma-distributed rates $(+\mathrm{R} \text { or }+\mathrm{G})^{71}$. Bootstrap support values were inferred from 1000

520 ultrafast bootstrap (ufBV) replicates and from 1000 replicates of the SH-like approximate

521 likelihood ratio test (SH-aLRT). All unprocessed phylogenetic trees can be found in

522 Supplementary Data 4.

\section{Phylogenetic inference of interspecies relationships within the Chlamydiae phylum}

524 Identification and phylogenetic analysis of $16 \mathrm{~S}$ rRNA gene amplicon and metagenome sequences

525 Barrnap $^{53} 0.8$ was used to identify $16 \mathrm{~S} / 18 \mathrm{~S}$ rRNA genes in the metagenomic assemblies, three 526 iterations with 'kingdom' set to 'euk', 'arc' and 'bac' were run, with 'reject' set to $20 \%$ of the

527 rRNA gene. Sequences were taxonomically classified using LCAClassifier ${ }^{37}$ from CREST-3.0.5

528 with silvamod128 as the reference database. The microbial composition of each metagenome can

529 be found in Supplementary Table 4. A reference dataset of near-full length chlamydiae $16 \mathrm{~S}$

530 rRNA gene sequences from various metagenomic and amplicon sequence databases ${ }^{6}$ was used

531 for determining the phylogenetic placement of these sequences. ML phylogenetic inference (Fig.

532 1c, Supplementary Fig. 2) was performed with the GTR+R7 model of evolution ${ }^{72}$ (based on

533 model selection).

\section{Selection of single-copy marker proteins}

535 We selected marker proteins using NOGs that were present in a single-copy in $95 \%$ of near536 complete PVC genomes (Supplementary Fig. 14). For each of the 149 markers protein initially

537 identified, alignments were generated and manually curated to remove divergent sequences prior 538 to final alignment and trimming with trimAl ${ }^{66} \mathrm{v} 1.4$ (--automated1). ML phylogenies were 539 inferred for each alignment, and proteins displaying patterns of vertical inheritance were selected. 
540 Due to strain microdiversity, it is not uncommon to have contigs from two different, but closely

541 related, lineages together in a MAG, resulting in redundancy. For each marker, in cases of

542 multiple copies from the same MAG, alignments and corresponding single-gene phylogenies

543 were manually inspected to determine if it represented a paralog, a redundant gene copy, or

544 partial sequences from the same gene. If redundant sequences overlapped with non-identical

545 regions, all sequences from the same genome were removed; if they were placed at the end of a

546 contig and shared an identical overlapping region (longer than 30 nucleotides), the sequences

547 were merged; and if they were partial non-overlapping protein fragments, the longer fragment

548 was selected and the shorter one removed. This inspection resulted in 126 remaining protein

549 markers which were subjected to discordance filtering (or " $\chi^{2}$ trimming") to remove markers with

550 the most conflicting phylogenetic signal ${ }^{73}$. The $20 \%$ of markers whose taxon bipartition profiles

551 were least concordant with the others, resulting in a high discordance score (Supplementary Fig.

552 15), were removed. This final set of 98 marker proteins was concatenated and used for species-

553 tree reconstruction (Supplementary Table 8).

$554 \quad$ Phylogenetic inference of interspecies relationships using concatenated marker proteins

555 Chlamydial species representatives (released prior to February $6^{\text {th }}, 2017$, Supplementary Table 3 )

556 and marine sediment chlamydiae MAGs (Supplementary Table 2) were used to investigate

557 interspecies phylogenomic relations within the Chlamydiae phylum, using other PVC

558 representatives as an outgroup (Supplementary Table 6). Marker proteins were separately aligned

559 and trimmed before being concatenated into a supermatrix. ML inference was performed on a

560 supermatrix of all 98 identified single-copy marker proteins (28,286 amino acid positions), and a

561 sub-selection of the 55 (14,212 amino acid positions) and 38 (7,894 amino acid positions)

562 markers with the highest representation among lineages (Supplementary Table 8), using the

$563 \mathrm{LG}+\mathrm{C} 60+\mathrm{G}+\mathrm{F}$ model of evolution. The tree topologies inferred were similar across all three 
564 datasets (Supplementary Fig. 16), indicating that the 38 marker protein sub-selection was

565 sufficient for further inferences. Several more in-depth phylogenetic analyses were applied to the

566 supermatrix of 38 marker proteins (Fig. 2), using a smaller outgroup to allow for more

567 computationally intensive analyses (Supplementary Table 6). PMSF is a site-heterogeneous

568 mixture model that can closely approximate complex mixture models such as $\mathrm{LG}+\mathrm{C} 60+\mathrm{G}+\mathrm{F}$

569 while reducing computational time several-fold ${ }^{74}$, making full bootstrapping practical. A ML

570 phylogeny was inferred using the PMSF model implemented in IQ-TREE ${ }^{67} 1.5 .5$, with a guide-

571 tree inferred using the $\mathrm{LG}+\mathrm{C} 60+\mathrm{G}+\mathrm{F}$ model of evolution, with 100 nonparametric bootstrap

572 replicates $(\mathrm{BV})$. The same analysis was performed on the alignment, after it was subjected to $\chi^{2}-$

573 trimming. Here, the proportion of most heterogeneous sites were was removed in a step-wise

574 fashion from $5 \%$ to $95 \%$ of sites, as previously described ${ }^{48,75}$. The resulting $\chi^{2}$ test statistics for

575 each lineage under the various heterogenous site removal treatments were visualized for the

576 chlamydiae and PVC outgroup (Supplementary Fig. 17) and 25\% removal was chosen as the

577 treatment which best lowered compositional heterogeneity while retaining the largest number of

578 informative sites. Bayesian analysis of this alignment was performed under the CAT $+\mathrm{GTR}+\Gamma 4$

579 model with PhyloBayes MPI ${ }^{76}$ 1.7a. Four independent Markov chain Monte Carlo chains were

580 run for $\sim 55,000$ generations, after which, three chains converged (maxdiff $=0.12$; burn-in $=$

581 15,000). Subsequently, these 38 markers were updated with additional Chlamydiae species

582 representatives (released between February $6^{\text {th }}, 2017$ and April $18^{\text {th }}, 2018$, Supplementary Table

583 3). Single-protein phylogenies and alignments including these lineages were inferred and

584 manually inspected, as described above, followed by concatenation of each of the 38 trimmed

585 alignments. A robust ML phylogeny was inferred based on the resulting supermatrix using the

586 selected LG+C60+PMSF model, as described above (Supplementary Fig. 3). 
589 Sequences identified for each NF-T3SS/flagellar gene (see above) were gathered, separately

590 aligned and trimmed with $\mathrm{BMGE}^{77}$ v. 1.12 (-m BLOSUM30). ML phylogenies were inferred

591 with IQ-TREE ${ }^{67}$ v1.6.5 with model selection among the following models: the empirical LG

592 model $^{69}$ the empirical profile mixture models (C10 to $\left.\mathrm{C} 60\right)$ combined with the LG

593 exchangeability matrix (e.g., $\mathrm{LG}+\mathrm{C} 10)^{70}$, with or without empirically determined amino acid

594 frequencies $(+\mathrm{F})$, and specific free rates $(+\mathrm{R} 0,+\mathrm{R} 2,+\mathrm{R} 4 \text { or }+\mathrm{R} 6)^{71}$. An additional $\mathrm{ML}$

595 reconstruction was run with the same alignments using the $\mathrm{PMSF}^{74}$ approximation of the selected

596 model and the previously obtained tree as a guide tree, with $100 \mathrm{BV}$. These reconstructions were

597 used to reclassify sequences between the NF-T3SS/flagellar homologues and outgroups.

598 Phylogenies of individual components were largely congruent (Supplementary Data 4), allowing

599 us to reconstruct a concatenated phylogeny using the separately trimmed alignments of the sctJ,

$600 s c t N, \quad s c t R, \quad s c t S, \quad s c t T, \quad s c t U$, and $s c t V$ homologues with IQ-TREE ${ }^{67}$ v1.6.5 as above

601 (Supplementary Data 4).

$602 \quad$ Nucleotide transporters

603 The diversity and phylogenetic placement of nucleotide transporter (NTT) proteins from marine

604 sediment chlamydiae was investigated. The region corresponding to the NTT $\mathrm{PF}^{78}$ domain 605 (PF03219) with a single-domain structure was predicted using hmmscan from the HMMER v3.1

606 toolkit (http://hmmer.org) and extracted from the full protein sequences. An initial phylogeny

607 (not shown) was reconstructed based on this conserved region using FastTree ${ }^{79}$, and indicated

608 two monophyletic clades separated by a long branch as identified previously ${ }^{80}$. Further

609 phylogenetic analyses were performed separately on the clade containing "canonical NTT" and

610 the "other NTT" clade (Supplementary Fig. 12). NTT proteins containing a HEAT domain were

611 also investigated (Supplementary Fig. 12). Proteins homologous to the Chlamydia trachomatis 
612 query were retrieved from marine sediment chlamydiae using BLASTP and were combined with

613 NTT-HEAT sequences identified in a prior study ${ }^{80}$. All three NTT datasets were each aligned

614 before being trimmed with TrimAl ${ }^{81} \mathrm{v} 1.4$, using the 'gappyout' option for the "canonical NTT"

615 alignment and the 'automated1' option for the "sister NTT" and NTT-HEAT alignments. The

$616 \mathrm{LG}+\mathrm{F}+\mathrm{R} 8$ model of evolution was selected for ML phylogenetic inference, except for the NTT-

617 HEAT alignments, where the LG+F+R6 model was selected instead. Chlamydiae NTTs that have

618 been functionally characterized were annotated in the resulting phylogenies (Supplementary Fig.

619 12, Supplementary Discussion).

620 Proteins conserved in CC-IV and Chlamydiaceae

$621 \mathrm{NOGs}^{62}$ and $\mathrm{PF}^{78}$ domains identified uniquely in clade CC-IV and Chlamydiaceae among 622 chlamydial lineages (Supplementary Table 3) were compiled (Supplementary Fig. 6 and 7) and

623 phylogenetic investigation of these sequences with the PF domains PF04518 and PF05302

624 performed (Supplementary Fig. 7). Hmmalign, from the HMMER v3.1 toolkit

625 (http://hmmer.org), was used to extract the region corresponding to the domains from sequences,

626 which were aligned and trimmed as outlined previously. ML phylogenies were inferred using the

627 PMSF model implemented in IQ-TREE ${ }^{67}$ 1.5.5, with a guide-tree inferred using the

$628 \mathrm{LG}+\mathrm{C} 20+\mathrm{G}+\mathrm{F}$ model of evolution, with $100 \mathrm{BV}$. An hmmsearch against the $n r$ database

629 confirmed that these PF domains are only present in CC-IV and Chlamydiaceae chlamydiae.

\section{Determination of replication rates}

631 We used $\operatorname{RRep}^{24}$ to determine the replication rate of the microbial population represented by each

632 MAG. The tool uses differences in sequencing coverage that arise bi-directionally across the 633 genomes of replicating bacteria, due to the single origin of replication, to infer a population-level 634 rate of replication ${ }^{24}$. Since iRep calculations require MAGs that are estimated to be $75 \%$ 635 complete with $5 \mathrm{X}$ coverage, we analyzed only 15 of the 24 MAGs that met these criteria. 
636 Sequenced reads from each metagenome were mapped to corresponding assembled contigs using

637 Bowtie $2^{51}$ with the 'reorder' option, before applying iRep ${ }^{24}$ v.1.10 with default settings (Fig. 4a).

\section{Chlamydial environmental diversity}

639 Using the Integrated Microbial NGS platform (IMNGS) ${ }^{82}$ (accessed March $5^{\text {th }}, 2018$ ), which 640 systematically screens prokaryotic $16 \mathrm{~S}$ rRNA gene amplicon datasets deposited as sequence read 641 archives in NCBI, the percentage of samples from select environments with a relative abundance 642 of over $0.1 \%$ Chlamydiae, and a percentage with at least 50 OTUs was assessed (Fig. 4b).

\section{Data visualization}

644 Plots in figures were made with R v.3.2.2 (R Development Core Team, 2008) using the packages 645 ggplot $^{83}$ and gplots ${ }^{84}$. NOG absence and presence profiles across chlamydial genomes were 646 evaluated using top NOG hits identified by eggNOG mapper ${ }^{63}$ as described above 647 (Supplementary Data 3). A binary distance matrix of NOG presence patterns was hierarchically 648 clustered using hclust with the 'average' agglomeration method in R, and a heatmap generated 649 using heatmap. 2 from the gplots ${ }^{84}$ package (Supplementary Fig. 3). Intersection plots were 650 implemented using the R package $\operatorname{UpSetR}^{85}$ (Fig. 3b). Synteny figures for NF-T3SS/flagellar 651 systems (Supplementary Figs. 8, 9, 10 and 11) were generated using the R package GenoPlotR ${ }^{86}$. 652 Phylogenetic trees were visualized and edited using Figtree ${ }^{87}$ v1.4.2 and iTOL ${ }^{88}$. Protein domains 653 were visualized and mapped to phylogenetic trees using iTOL ${ }^{88}$. Bathymetry was uploaded and 654 visualized using GeoMapApp V. 3.6.6 (http://www.geomapapp.org). Figures were made and 655 edited using Inkscape and Adobe Illustrator.

656 Data availability

657 Raw sequence reads for both 16S rRNA gene amplicons and metagenomes have been deposited 658 to the NCBI Sequence Read Archive repository under BioProject PRJNA504765. Whole

659 Genome Shotgun projects for metagenome assemblies GS08_GC12_126, GS10_PC15_940, 
GS10_PC15_1000 and GS10_PC15_1060 have been deposited at DDBJ/ENA/GenBank under

the accessions SDBU00000000, SDBV00000000, SDBS00000000 and SDBT00000000,

662

respectively. The versions described in this paper are versions SDBU01000000, SDBV01000000,

663 SDBS01000000 and SDBT01000000. Accessions for MAGs generated in this study can be found

664 in Supplementary Table 2, and are linked to BioProject PRJNA504765. Files containing

665 sequence datasets, alignments, all 16S rRNA gene amplicon OTUs and additional data generated

666 in this study are archived at the Dryad Digital Repository: https://datadryad.org/resource/XXX.

\section{Method References}

670

671

672

673

674

675

676

677

678

679

680

681

682

683

684

685

686

687

688

689

690

691

692

693

694

695

696

26 Jorgensen, S. L. et al. Correlating microbial community profiles with geochemical data in highly stratified sediments from the Arctic Mid-Ocean Ridge. PNAS 109, E2846-E2855, doi:10.1073/pnas.1207574109 (2012).

27 Jorgensen, S. L., Thorseth, I. H., Pedersen, R. B., Baumberger, T. \& Schleper, C. Quantitative and phylogenetic study of the Deep Sea Archaeal Group in sediments of the Arctic mid-ocean spreading ridge. Front Microbiol 4, 299, doi:10.3389/fmicb.2013.00299 (2013).

28 Hugenholtz, P., Pitulle, C., Hershberger, K. 1. \& Pace, N. r. Novel Division Level Bacterial Diversity in a Yellowstone Hot Spring. Journal of Bacteriology 180, 366-376 (1998).

29 Klindworth, A. et al. Evaluation of general 16S ribosomal RNA gene PCR primers for classical and next-generation sequencing-based diversity studies. Nucleic Acids Res 41, e1, doi:10.1093/nar/gks808 (2013).

30 Hugerth, L. W. et al. Systematic design of $18 \mathrm{~S}$ rRNA gene primers for determining eukaryotic diversity in microbial consortia. PLoS One 9, e95567, doi:10.1371/journal.pone.0095567 (2014).

31 Ashelford, K. E., Weightman, A. J. \& Fry, J. C. PRIMROSE: a computer program for generating and estimating the phylogenetic range of $16 \mathrm{~S}$ rRNA oligonucleotide probes and primers in conjunction with the RDP-II database. Nucleic Acids Res 30, 3481-3489 (2002).

32 Acinas, S. G., Sarma-Rupavtarm, R., Klepac-Ceraj, V. \& Polz, M. F. PCR-induced sequence artifacts and bias: insights from comparison of two 16S rRNA clone libraries constructed from the same sample. Appl Environ Microbiol 71, 8966-8969, doi:10.1128/AEM.71.12.8966-8969.2005 (2005).

33 Martin, M. Cutadapt removes adapter sequences from high-throughput sequencing reads. EMBnet.Journal 17, 10-12, doi:10.14806/ej.17.1.200 (2011). 
Rognes, T., Flouri, T., Nichols, B., Quince, C. \& Mahe, F. VSEARCH: a versatile open source tool for metagenomics. PeerJ 4, e2584, doi:10.7717/peerj.2584 (2016).

35 Edgar, R. C., Haas, B. J., Clemente, J. C., Quince, C. \& Knight, R. UCHIME improves sensitivity and speed of chimera detection. Bioinformatics 27, 2194-2200, doi:10.1093/bioinformatics/btr381 (2011).

Quast, C. et al. The SILVA ribosomal RNA gene database project: improved data processing and web-based tools. Nucleic Acids Res 41, D590-596, doi:10.1093/nar/gks1219 (2013). One 7, e49334, doi:10.1371/journal.pone.0049334 (2012). sequence data. Bioinformatics 30, 2114-2120, doi:10.1093/bioinformatics/btu170 (2014). Andrews, S. FastQC: A quality control tool for high throughput sequence data. (2010). Gurevich, A., Saveliev, V., Vyahhi, N. \& Tesler, G. QUAST: quality assessment tool for genome assemblies. Bioinformatics 29, 1072-1075, doi:10.1093/bioinformatics/btt086 (2013).

41 Hyatt, D. et al. Prodigal: prokaryotic gene recognition and translation initiation site identification. BMC Bioinformatics 11, 119, doi:10.1186/1471-2105-11-119 (2010).

42 Nomura, M. \& Morgan, E. A. Genetics of bacterial ribosomes. Annual Reviews Genetics 11, 297-347 (1977).

43 Martijn, J., Vosseberg, J., Guy, L., Offre, P. \& Ettema, T. J. G. Deep mitochondrial origin outside the sampled alphaproteobacteria. Nature 557, 101-105, doi:10.1038/s41586-0180059-5 (2018).

44 Raymann, K., Brochier-Armanet, C. \& Gribaldo, S. The two-domain tree of life is linked to a new root for the Archaea. Proc Natl Acad Sci U S A 112, 6670-6675, doi:10.1073/pnas.1420858112 (2015).

45 Bray, N. L., Pimentel, H., Melsted, P. \& Pachter, L. Near-optimal probabilistic RNA-seq quantification. Nat Biotechnol 34, 525-527, doi:10.1038/nbt.3519 (2016).

46 Alneberg, J. et al. Binning metagenomic contigs by coverage and composition. Nat Methods 11, 1144-1146, doi:10.1038/nmeth.3103 (2014).

47 Hugerth, L. W. et al. Metagenome-assembled genomes uncover a global brackish microbiome. Genome Biol 16, 279, doi:10.1186/s13059-015-0834-7 (2015).

48 Martijn, J. et al. Single-cell genomics of a rare environmental alphaproteobacterium provides unique insights into Rickettsiaceae evolution. ISME $J$ 9, 2373-2385, doi:10.1038/ismej.2015.46 (2015).

49 Karst, S. M., Kirkegaard, R. H. \& Albertsen, M. mmgenome: a toolbox for reproducible genome extraction from metagenomes. bioRxiv, doi:10.1101/059121 (2016).

50 Bowers, R. M. et al. Minimum information about a single amplified genome (MISAG) and a metagenome-assembled genome (MIMAG) of bacteria and archaea. Nat Biotechnol 35, 725-731, doi:10.1038/nbt.3893 (2017).

51 Langmead, B. \& Salzberg, S. L. Fast gapped-read alignment with Bowtie 2. Nat Methods 9, 357-359, doi:10.1038/nmeth.1923 (2012).

52 Stamatakis, A. RAxML version 8: a tool for phylogenetic analysis and post-analysis of large phylogenies. Bioinformatics 30, 1312-1313, doi:10.1093/bioinformatics/btu033 (2014).

53 Seemann, T. Prokka: rapid prokaryotic genome annotation. Bioinformatics 30, 20682069, doi:10.1093/bioinformatics/btu153 (2014). 
776

777

778

779

780

781

782

783

784

785

786

787

788

789

790

54 Buchfink, B., Xie, C. \& Huson, D. H. fast and sensitive protein alignment using DIAMOND. Nature Methods 12, 59-60, doi:10.1038/nmeth.3176 (2015).

55 Finn, R. D. et al. Pfam: the protein families database. Nucleic Acids Res 42, D222-230, doi:10.1093/nar/gkt1223 (2014).

56 Finn, R. D. et al. InterPro in 2017-beyond protein family and domain annotations. Nucleic Acids Res 45, D190-D199, doi:10.1093/nar/gkw1107 (2017).

57 Caspi, R. et al. The MetaCyc database of metabolic pathways and enzymes and the BioCyc collection of Pathway/Genome Databases. Nucleic Acids Res 42, D459-471, doi:10.1093/nar/gkt1103 (2014).

58 Kanehisa, M., Sato, Y., Kawashima, M., Furumichi, M. \& Tanabe, M. KEGG as a reference resource for gene and protein annotation. Nucleic Acids Res 44, D457-462, doi:10.1093/nar/gkv1070 (2016).

59 Kanehisa, M. \& Goto, S. KEGG: Kyoto Encyclopedia of Genes and Genomes. Nucleic Acids Research 28, 27-30 (2000).

60 Jones, P. et al. InterProScan 5: genome-scale protein function classification. Bioinformatics 30, 1236-1240, doi:10.1093/bioinformatics/btu031 (2014).

61 Kanehisa, M., Sato, Y. \& Morishima, K. BlastKOALA and GhostKOALA: KEGG Tools for Functional Characterization of Genome and Metagenome Sequences. J Mol Biol 428, 726-731, doi:10.1016/j.jmb.2015.11.006 (2016).

62 Huerta-Cepas, J. et al. eggNOG 4.5: a hierarchical orthology framework with improved functional annotations for eukaryotic, prokaryotic and viral sequences. Nucleic Acids Res 44, D286-293, doi:10.1093/nar/gkv1248 (2016).

63 Huerta-Cepas, J. et al. Fast Genome-Wide Functional Annotation through Orthology Assignment by eggNOG-Mapper. Mol Biol Evol 34, 2115-2122, doi:10.1093/molbev/msx148 (2017).

64 Abby, S. S. et al. Identification of protein secretion systems in bacterial genomes. Sci Rep 6, 23080, doi:10.1038/srep23080 (2016).

65 Eichinger, V. et al. EffectiveDB--updates and novel features for a better annotation of bacterial secreted proteins and Type III, IV, VI secretion systems. Nucleic Acids Res 44, D669-674, doi:10.1093/nar/gkv1269 (2016).

66 Capella-Gutiérrez, S., Silla-Martínez, J. M. \& Gabaldón, T. trimAl: a tool for automated alignment trimming in large-scale phylogenetic analyses. Bioinformatics 25, 1972-1973 (2009).

67 Nguyen, L. T., Schmidt, H. A., von Haeseler, A. \& Minh, B. Q. IQ-TREE: a fast and effective stochastic algorithm for estimating maximum-likelihood phylogenies. Mol Biol Evol 32, 268-274, doi:10.1093/molbev/msu300 (2015).

68 Kalyaanamoorthy, S., Minh, B. Q., Wong, T. K. F., von Haeseler, A. \& Jermiin, L. S. ModelFinder: fast model selection for accurate phylogenetic estimates. Nat Methods 14, 587-589, doi:10.1038/nmeth.4285 (2017).

69 Le, S. Q. \& Gascuel, O. An improved general amino acid replacement matrix. Mol Biol Evol 25, 1307-1320, doi:10.1093/molbev/msn067 (2008).

70 Quang le, S., Gascuel, O. \& Lartillot, N. Empirical profile mixture models for $\begin{array}{llll}\text { phylogenetic } & \text { reconstruction. } & \text { 24ioinformatics }\end{array}$ doi:10.1093/bioinformatics/btn445 (2008).

71 Le, S. Q., Dang, C. C. \& Gascuel, O. Modeling Protein Evolution with Several Amino Acid Replacement Matrices Depending on Site Rates. Molecular Biology and Evolution 29, 2921-2936, doi:10.1093/molbev/mss112 (2012). 
72 Tavaré, S. Some probabilistic and statistical problems in the analysis of DNA sequences. Lectures on mathematics in the life sciences 17, 57-86 (1986).

73 Williams, K. P. et al. Phylogeny of gammaproteobacteria. J Bacteriol 192, 2305-2314, doi:10.1128/JB.01480-09 (2010).

74 Wang, H. C., Minh, B. Q., Susko, E. \& Roger, A. J. Modeling Site Heterogeneity with Posterior Mean Site Frequency Profiles Accelerates Accurate Phylogenomic Estimation. Syst Biol 67, 216-235, doi:10.1093/sysbio/syx068 (2018).

75 Viklund, J., Ettema, T. J. \& Andersson, S. G. Independent genome reduction and phylogenetic reclassification of the oceanic SAR11 clade. Mol Biol Evol 29, 599-615, doi:10.1093/molbev/msr203 (2012).

76 Lartillot, N., Rodrigue, N., Stubbs, D. \& Richer, J. PhyloBayes MPI: phylogenetic reconstruction with infinite mixtures of profiles in a parallel environment. Syst Biol 62, 611-615, doi:10.1093/sysbio/syt022 (2013).

77 Criscuolo, A. \& Gribaldo, S. BMGE (Block Mapping and Gathering with Entropy): a new software for selection of phylogenetic informative regions from multiple sequence alignments. BMC Evolutionary Biology 10, doi:https://doi.org/10.1186/1471-2148-10-210 (2010).

78 Finn, R. D. et al. The Pfam protein families database: towards a more sustainable future. Nucleic Acids Res 44, D279-285, doi:10.1093/nar/gkv1344 (2016).

79 Price, M. N., Dehal, P. S. \& Arkin, A. P. FastTree 2 - Approximately MaximumLikelihood Trees for Large Alignments. PLoS one 5, doi:doi:10.1371/ journal.pone.0009490 (2010).

80 Major, P., Embley, T. M. \& Williams, T. A. Phylogenetic Diversity of NTT Nucleotide Transport Proteins in Free-Living and Parasitic Bacteria and Eukaryotes. Genome Biol Evol 9, 480-487, doi:10.1093/gbe/evx015 (2017).

81 Capella-Gutiérrez, S., Silla-Martínez, J. M. \& Gabaldón, T. trimAl: a tool for automated alignment trimming in large-scale phylogenetic analyses. Bioinformatics 25, 1972-1973 (2009).

82 Lagkouvardos, I. et al. IMNGS: A comprehensive open resource of processed 16S rRNA microbial profiles for ecology and diversity studies. Sci Rep 6, 33721, doi:10.1038/srep33721 (2016).

83 Wickham, H. ggplot2: Elegant Graphics for Data Analysis. Springer-Verlag New York (2009).

84 Gregory R Warnes, B. B., Lodewijk Bonebakker, Robert Gentleman, Wolfgang Huber, Andy Liaw, Thomas Lumley, Martin Maechler, Arni Magnusson, Steffen Moeller, Marc Schwartz, Bill Venables. gplots: Various $\mathrm{R}$ programming tools for plotting data. $R$ package version (2009).

85 Lex, A., Gehlenborg, N., Strobelt, H., Vuillemot, R. \& Pfister, H. UpSet: Visualization of Intersecting Sets. IEEE Trans Vis Comput Graph 20, 1983-1992, doi:10.1109/TVCG.2014.2346248 (2014).

86 Guy, L., Roat Kultima, J. \& Andersson, S. G. E. genoPlotR: comparative gene and genome visualization in R. Bioinformatics 26, 2334-2335, doi:10.1093/bioinformatics/btq413 (2010).

87 Rambaut, A. FigTree v1.3.1. Institute of Evolutionary Biology, University of Edinburgh, Edinburgh., doi:http://tree.bio.ed.ac.uk/software/figtree/ (2010). 
bioRxiv preprint doi: https://doi.org/10.1101/577767. this version posted March 16, 2019. The copyright holder for this preprint (which was not certified by peer review) is the author/funder. It is made available under a CC-BY-NC-ND 4.0 International license.

$83688 \quad$ Letunic, I. \& Bork, P. Interactive tree of life (iTOL) v3: an online tool for the display and annotation of phylogenetic and other trees. Nucleic Acids Res 44, W242-245, doi:10.1093/nar/gkw290 (2016). 